\title{
Aptamer fluorescence anisotropy sensors for adenosine triphosphate by comprehensive screening tetramethylrhodamine labeled nucleotides
}

\author{
Qiang Zhao ${ }^{\mathrm{a}, \mathrm{b}, *}$, Qin Lv $^{\mathrm{a}}$, Hailin Wang ${ }^{\mathrm{b}}$ \\ ${ }^{a}$ Institute of Environmental Science, College of Chemistry and Chemical Engineering, Shanxi University, Taiyuan 030006, PR China \\ ${ }^{\mathrm{b}}$ State Key Laboratory of Environmental Chemistry and Ecotoxicology, Research Center for Eco-Environmental Sciences, Chinese Academy of Sciences, \\ Beijing 100085, PR China
}

\section{A R T I C L E I N F O}

\section{Article history:}

Received 2 February 2015

Received in revised form

13 March 2015

Accepted 15 March 2015

Available online 17 March 2015

\section{Keywords:}

Aptamer

Fluorescence anisotropy

Fluorescence polarization

Adenosine triphosphate

Fluorescence sensor

Labeling site

\begin{abstract}
A B S T R A C T
We previously reported a fluorescence anisotropy (FA) approach for small molecules using tetramethylrhodamine (TMR) labeled aptamer. It relies on target-binding induced change of intramolecular interaction between TMR and guanine (G) base. TMR-labeling sites are crucial for this approach. Only terminal ends and thymine (T) bases could be tested for TMR labeling in our previous work, possibly causing limitation in analysis of different targets with this FA strategy. Here, taking the analysis of adenosine triphosphate (ATP) as an example, we demonstrated a success of conjugating TMR on other bases of aptamer adenine (A) or cytosine (C) bases and an achievement of full mapping various labeling sites of aptamers. We successfully constructed aptamer fluorescence anisotropy (FA) sensors for adenosine triphosphate (ATP). We conjugated single TMR on adenine (A), cytosine (C), or thymine (T) bases or terminals of a 25-mer aptamer against ATP and tested FA responses of 14 TMR-labeled aptamer to ATP. The aptamers having TMR labeled on the 16th base $C$ or 23rd base A were screened out and exhibited significant FA-decreasing or FA-increasing responses upon ATP, respectively. These two favorable TMRlabeled aptamers enabled direct FA sensing ATP with a detection limit of $1 \mu \mathrm{M}$ and the analysis of ATP in diluted serum. The comprehensive screening various TMR labeling sites of aptamers facilitates the successful construction of FA sensors using TMR-labeled aptamers. It will expand application of TMR-G interaction based aptamer FA strategy to a variety of targets.
\end{abstract}

(c) 2015 Elsevier B.V. All rights reserved.

\section{Introduction}

Aptamers are single stranded nucleic acid selected in evolutionary process binding to targets with good specificity and binding affinity (Citartan et al., 2012; Feng et al., 2014; Juskowiak, 2011; Kim and Gu 2014; Li et al., 2015; Liu et al., 2009; McKeague and Derosa, 2012). Since the discovery of aptamers in 1990s, aptamer-based assays for small molecules have drawn increasing attentions in environmental sensing, food safety, and clinical analysis due to the unique features of aptamers, such as easy generation, facile labeling, good thermal stability, small size, and target-binding induced structure change (Citartan et al., 2012; Feng et al., 2014; Juskowiak, 2011; Kim and Gu 2014; Li et al., 2015; Liu et al., 2009; McKeague and Derosa, 2012). Taking advantage of fluorescence anisotropy (FA) or fluorescence polarization (FP) in

\footnotetext{
* Corresponding author at: Institute of Environmental Science, College of Chemistry and Chemical Engineering, Shanxi University, Taiyuan 030006, PR China. Fax: + 863517011011 .

E-mail address: chemzhaoq@hotmail.com (Q. Zhao).
}

sensitivity, reproducibility, and simplicity (Lea and Simeonov, 2011; Gradinaru et al., 2010; Le et al., 2002; Smith and Eremin, 2008), the aptamer-based FA/FP sensors are attractive (Gokulrangan et al., 2005; Liu et al., 2009; Ruta et al., 2009; Zhang et al., 2011).

In spite of ease of analyzing proteins (Gokulrangan et al., 2005; Zhang et al., 2011), the dye-labeled aptamer based direct FA sensors remain challenging for small molecule analysis because the binding of small target only brings negligible mass change of aptamer. Under a rational design, aptamers with dye labeled on terminal ends can be used to direct detection of small molecules due to structureswitch induced FA alteration (Kidd et al., 2011; Perrier et al., 2010; Ruta et al., 2009), but the produced FA changes are usually mild. To improve FA/FP signals in small molecule detection, large biomolecules (e.g. proteins and oligonucleotides) or nanomaterials (e.g. gold nanoparticles, graphene, and silica nanoparticles) have been introduced in assay development by increasing the binding-induced molecular weight change (Cruz-Aguado and Penner, 2008; Cui et al., 2012; Huang et al., 2012; Liu et al., 2013; Ye, and Yin 2008; Yu et al., 2013; Zhu et al., 2011, 2012). 
Recently, we have reported a simple and noncompetitive FA strategy for a small molecule, ochratoxin A, by using a TMR-labeled aptamer. (Zhao et al., 2014) It is based on the target-binding induced change of intramolecular interactions between TMR and the guanine $(G)$ bases of the aptamer. The interaction significantly affects local rotation of TMR and FA of TMR. In this FA strategy, TMR-labeling position of the aptamers plays a crucial role. The intramolecular interaction between TMR and G bases is distance dependent, and TMR labeling may influence the binding affinity of aptamer depending on the labeling sites (Zhao et al., 2014). An ideal labeling position allows TMR-labeled aptamer showing FA change upon target without loss in binding affinity. Previously, only terminal ends and $\mathrm{T}$ base of aptamers could be examined for TMR labeling in FA analysis of ochratoxin A (Zhao et al., 2014). However, $G$ base in aptamers may not be surrounded by $T$ bases or terminal ends and some aptamers may have less $\mathrm{T}$ bases. In addition, the binding affinity of aptamer may be disrupted by introducing TMR on those limited labeling sites. Therefore, lack of labeling single TMR on other labeling sites may cause failure and limitation of FA sensors using TMR-labeled aptamer. Achieving conjugation of a single TMR on other bases like A and C in aptamer will allow a full mapping of labeling positions of aptamers and overcome the limitation.

Herein, we successfully built a TMR-labeled aptamer FA sensor for adenosine triphosphate (ATP), an important compound in cell biology and biochemistry (Knowles, 1980), by comprehensive mapping the labeling sites of 5' terminal, 3' terminal, and the A, C, and $T$ bases of aptamers. We introduced a single TMR dye on various labeling sites of a 25-mer anti-ATP aptamer (Huizenga and Szostak, 1995; Lin and Patel, 1997), and screened FA response of each aptamer probe among 14 TMR-labeled aptamers on ATP. The aptamer with TMR labeled on the 16th base $C$ showed remarkable FA-decreasing response to ATP, while the aptamer with TMR labeled on the 23rd base A showed complementary and significant FA-increasing response to ATP. These aptamer probes enabled FA sensing ATP with detection limit of $1 \mu \mathrm{M}$ and detection of ATP in diluted serum samples. The achievement of conjugating TMR on A, $\mathrm{C}$, and $\mathrm{T}$ bases of aptamers can greatly facilitate the construction of FA sensor with TMR-labeled aptamer and expand the application of TMR-labeled aptamer FA sensor relying on the intramolecular interaction of TMR and $G$ base to the analysis of a variety of targets.

\section{Materials and methods}

\subsection{Chemical and reagents}

Adenosine triphosphate (ATP) was ordered from Sigma. All the DNA oligonucleotides were synthesized and purified by Takara Biotechnology (Dalian, China) or Sangon Biotech (Shanghai, China). Guanosine, thymidine, and cytidine were purchased from Amresco. Guanosine triphosphate (GTP), cytidine triphosphate (CTP), and uridine triphosphate (UTP) were ordered from Takara Biotechnology (Dalian, China). The 25-mer DNA aptamer against ATP had the following sequence: 5'-CCT GGG GGA GTA TTG CGG AGG AAG G-3' (Huizenga and Szostak, 1995). DNA aptamers with single TMR labeled at different positions were listed in Table 1.

\subsection{Fluorescence anisotropy measurement}

JASCO FP-8300 fluorescence spectrometer (Japan) was used for FA measurement, which was equipped with a thermostat for precise temperature control. In FA analysis experiments, FA of TMR was measured with an excitation at $560 \mathrm{~nm}$ and an emission at $578 \mathrm{~nm}$ (Zhao et al., 2014). Slits for the excitation and the emission
Table 1

List of TMR-labeled aptamers with respect of labeling sites.

\begin{tabular}{ll}
\hline Name & Sequences \\
\hline 5'-TMR-A25 & \\
1C TMR-A25 & 5'-CCT GGG GGA GTA TTG CGG AGG AAG G-3' \\
2C TMR-A25 & 5'-CCT GGG GGA GTA TTG CGG AGG AAG G-3' \\
3T TMR-A25 & 5'-CCT GGG GGA GTA TTG CGG AGG AAG G-3' \\
9A TMR-A25 & 5'-CCT GGG GGA GTA TTG CGG AGG AAG G-3' \\
11T TMR-A25 & 5'-CCT GGG GGA GTA TTG CGG AGG AAG G-3' \\
12A TMR-A25 & 5'-CCT GGG GGA GTA TTG CGG AGG AAG G-3' \\
13T TMR-A25 & 5'-CCT GGG GGA GTA TTG CGG AGG AAG G-3' \\
14T TMR-A25 & 5'-CCT GGG GGA GTA TTG CGG AGG AAG G-3' \\
16C TMR-A25 & 5'-CCT GGG GGA GTA TTG CGG AGG AAG G-3' \\
19A TMR-A25 & 5'-CCT GGG GGA GTA TTG CGG AGG AAG G-3' \\
22A TMR-A25 & 5'-CCT GGG GGA GTA TTG CGG AGG AAG G-3' \\
23A TMR-A25 & 5'-CCT GGG GGA GTA TTG CGG AGG AAG G-3' \\
3'-TMR-A25 & 5'-CCT GGG GGA GTA TTG CGG AGG AAG G-3' \\
\hline
\end{tabular}

a The bold characters show the labeling sites of TMR in the aptamer.

were both fixed at $5 \mathrm{~nm}$. In FA sensing ATP, TMR-labeled aptamer at $50 \mathrm{nM}$ was mixed with varying concentrations of ATP in a $200 \mu \mathrm{L}$ of buffer solution containing $50 \mathrm{mM}$ Tris- $\mathrm{HCl}(\mathrm{pH} \mathrm{7.5)}$, $50 \mathrm{mM} \mathrm{NaCl}, 20 \mathrm{mM} \mathrm{CaCl}_{2}$, and 0.1\% Tween 20, and then FA of TMR was measured after 10-min incubation. Without otherwise noted, FA measurement experiments were all performed at $20^{\circ} \mathrm{C}$.

\section{Results and discussion}

\subsection{Comprehensive screening FA responses of aptamers with TMR on} various labeling sites

Fig.1 shows the principle for FA sensing of ATP with TMR-labeled aptamers. The 25-nt anti-ATP aptamer contains $13 \mathrm{G}$ bases, 5A bases, 3C bases, and 4T bases (Huizenga and Szostak, 1995; Lin and Patel, 1997). Each G base is surrounded by different bases, experiencing distinct environment. The anti-ATP aptamer can form a hairpin structure when the aptamer binds to ATP, bringing a conformation change (Lin and Patel, 1997). The binding of ATP possibly alters the interaction between TMR and G bases, which affects FA of TMR (Heinlein et al., 2003; Unruh et al., 2005; Yabuki et al., 2003; Zhang et al., 2012), leading to FA changes of TMR. When ATP binding weakens TMR-G interaction, local rotation of labeled TMR increases. Thus, FA of TMR decreases with addition of ATP (Fig. 1a). In addition, when the affinity binding of ATP brings an enhanced TMR-G interaction, an increased FA may be observed

\section{a}

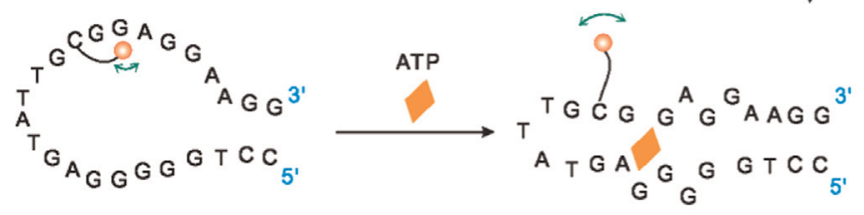

b
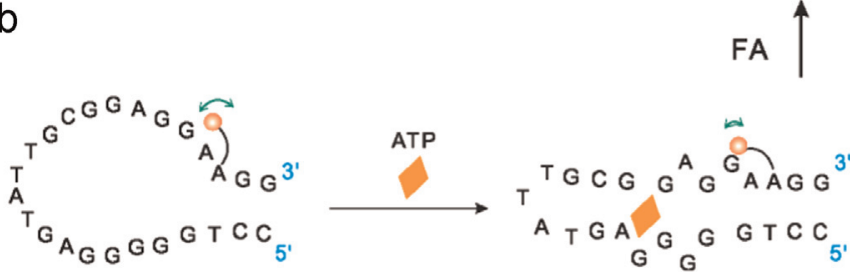

Fig. 1. Schematic diagram for detecting ATP by TMR-labeled aptamer sensor with FA-decreasing response (a) or FA-increasing response (b). 
as the strengthen interaction causes decreasing local rotation of TMR (Fig. 1b). To build a successful FA sensor for ATP with TMRlabeled aptamer, the TMR labeling site plays an important role. It is required that the labeled TMR interacts with $G$ bases in aptamer and target-binding induces a change of this interaction. In addition, to generate a good sensitivity in FA sensing for ATP, the binding affinity of the TMR-labeled aptamer needs to be well maintained. Therefore, mapping as many as possible labeling sites is highly demanded to obtain a TMR-labeled aptamer sensor with good FA responses.

We conjugated single TMR on $3^{\prime}$ end, $5^{\prime}$ end, A, C, or T bases of the aptamer against ATP (Zhang et al., 2011; Zhao et al., 2014), and obtained 14 different TMR-labeled aptamers (Table 1 and Fig. S1). Thus, we could make a complete screening FA responsive labeling site of aptamer. Fig. S1 in supplementary material shows how to conjugate TMR on different labeling sites of aptamer. The conjugation of TMR on A, C, or T base did not affect the labeled base stacking with the complementary base because the labeling did not occupy hydrogen bonding position, as Fig. S1 shows.

In absence of ATP, most of TMR-labeled aptamers exhibited FA value higher than 0.200 , while the 13T-TMR-A25 and 14T-TMRA25 showed FA values about 0.180 (Fig. 2A). As early studies show, the high FA value suggests a restricted local rotation of TMR resulted from intramolecular interaction between TMR and bases of aptamer, and the low FA value indicates a relatively free local rotation of labeled TMR (Gokulrangan et al., 2005; Ruta et al., 2009; Unruh et al., 2005; Zhao et al., 2014). As comparison, when TMR was conjugated on the 5 ' end of 25-nt poly T, 25-nt poly C or 25-nt poly A, the FA values of these TMR-labeled aptamers ranged from 0.100 to 0.160 . The results show the occurrence of intramolecular interaction between TMR and G bases in most of TMR-labeled aptamers against ATP.

We investigated the FA responses of fourteen TMR-labeled aptamers upon ATP. In presence of ATP, TMR-labeled aptamers showed significantly distinct responses to ATP. Fig. 2B shows the FA changes of the TMR-labeled aptamers ( $50 \mathrm{nM})$ caused by ATP at $100 \mu \mathrm{M}$. Fig. S2 in supplementary material shows FA responses of the TMR-labeled aptamers to varying concentrations of ATP. According to their FA responses to ATP, the TMR-labeled aptamers could be classified into three groups, the TMR-labeled aptamers with remarkable FA-increasing response upon ATP (22A-TMR-A25, 23A-TMR-A25, and 3'-TMR-A25), the TMR-labeled aptamers with remarkable FA-decreasing response upon ATP (2C-TMR-A25, 11TTMR-A25, 12A-TMR-A25, 13T-TMR-A25, 16C-TMR-A25, and 19ATMR-A25), and the TMR-labeled aptamers with little FA response upon ATP (5'-TMR-A25, 1C-TMR-A25, 3T-TMR-A25, 9A-TMR-A25, and 14T-TMR-A25). Comparing with the aptamers having TMR attached on $\mathrm{T}$ bases or terminal ends, the aptamer with TMR labeled on the 23rd base (A) of aptamer (23A-TMR-A25) gave the largest FA increase upon ATP. The aptamer with TMR labeling on the 16th base $C$ of aptamer (16C-TMR-A25) gave the largest FA decrease upon ATP. Without labeling TMR on A or C, it would be impossible to find the TMR-labeled aptamer with such a remarkable FA response to ATP. Clearly, comprehensive mapping various TMR labeling sites of aptamer greatly enhances the chances to build a FA responsive aptamer sensor. The full screening labeling sites overcomes the limitation of only mapping base $\mathrm{T}$ and terminal of aptamer in the previous study (Zhao et al., 2014). 23A-TMRA25 could be used to develop a FA sensor for ATP showing FAincrease response, and 16C-TMR-A25 could be used as a FA sensor for ATP showing FA-decrease response (Fig. 1).

\subsection{Optimization of FA sensing conditions}

We optimized the experimental conditions of FA sensing ATP with the 23A-TMR-A25 or 16C-TMR-A25 by testing the effects of

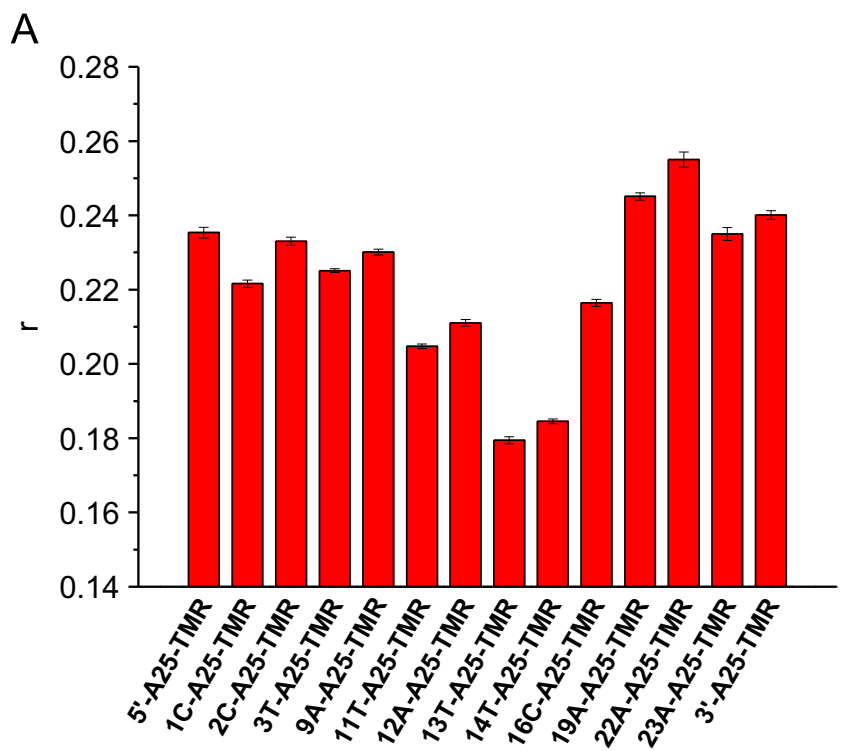

B

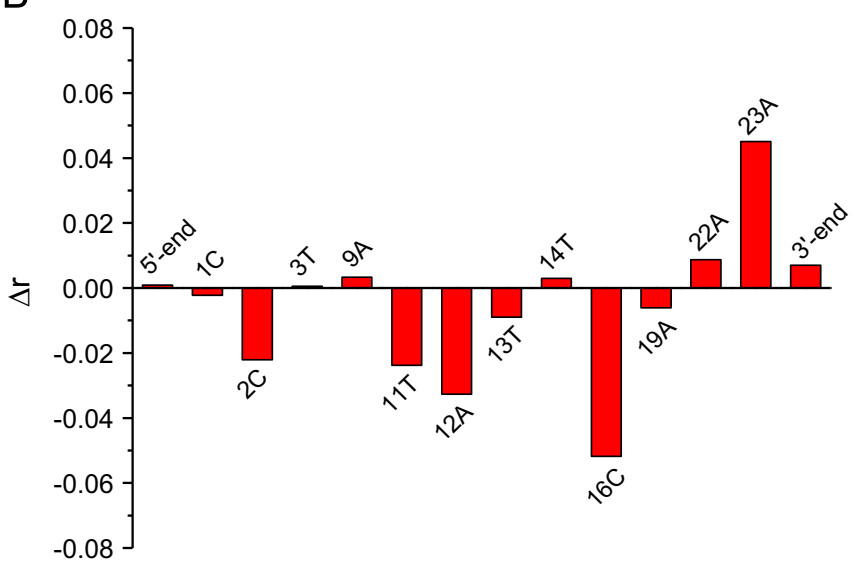

Fig. 2. FA values of TMR-labeled aptamers in absence of ATP (A) and FA changes of TMR-labeled aptamer upon ATP (B) with respect to labeling sites. The FA values of TMR-labeled aptamer and FA change of TMR-labeled aptamer ( $50 \mathrm{nM}$ ) caused by $100 \mu \mathrm{M}$ ATP were recorded in the buffer solution containing $50 \mathrm{mM}$ Tris- $\mathrm{HCl}(\mathrm{pH}$ 7.5), $50 \mathrm{mM} \mathrm{NaCl}, 20 \mathrm{mM} \mathrm{CaCl}_{2}$, and 0.1\% Tween 20 .

$\mathrm{NaCl}, \mathrm{CaCl}_{2}, \mathrm{pH}$, incubation temperature, and incubation time on the FA responses of TMR-labeled aptamer to ATP. For FA sensing of ATP with 23A-TMR-A25 probe, we assessed the effect of $\mathrm{NaCl}$ on the ATP induced-FA change by measuring the FA values of 23ATMR-A25 probe in absence of ATP and in presence of ATP $(100 \mu \mathrm{M})$ in the binding buffer solution containing varying concentrations of $\mathrm{NaCl}$, respectively (Fig. S3). With increase of concentration of $\mathrm{NaCl}$, the obtained FA change did not show remarkable alteration, and $50 \mathrm{mM} \mathrm{NaCl}$ in solution could give a little larger FA change than $\mathrm{NaCl}$ at concentrations higher than $50 \mathrm{mM}$. Solution containing $50 \mathrm{mM} \mathrm{NaCl}$ was applied for FA sensing ATP.

The presence of $\mathrm{CaCl}_{2}$ significantly affected the ATP-induced FA change of 23A-TMR-A25 (Fig. 3). The addition of $\mathrm{CaCl}_{2}$ decreased FA value of 23A-TMR-A25 in absence of ATP, suggesting $\mathrm{CaCl}_{2}$ may affect the local rotation of TMR in 23A-TMR-A25. In absence of $\mathrm{CaCl}_{2}, 23 \mathrm{~A}-\mathrm{TMR}-\mathrm{A} 25$ showed little FA response to ATP. With addition of $\mathrm{CaCl}_{2}$, 23A-TMR-A25 showed increasing FA responses to ATP, and highest FA increase was obtained at $20 \mathrm{mM} \mathrm{CaCl}_{2}$. Further increase of $\mathrm{CaCl}_{2}$ led to slight decrease of FA response of 23A-TMRA25 upon ATP. The result shows that $\mathrm{CaCl}_{2}$ has more effect on FA sensing ATP with 23A-TMR-A25 than $\mathrm{NaCl}$. $\mathrm{CaCl}_{2}$ is probably required for affinity binding between aptamer and ATP (Deng et al., 

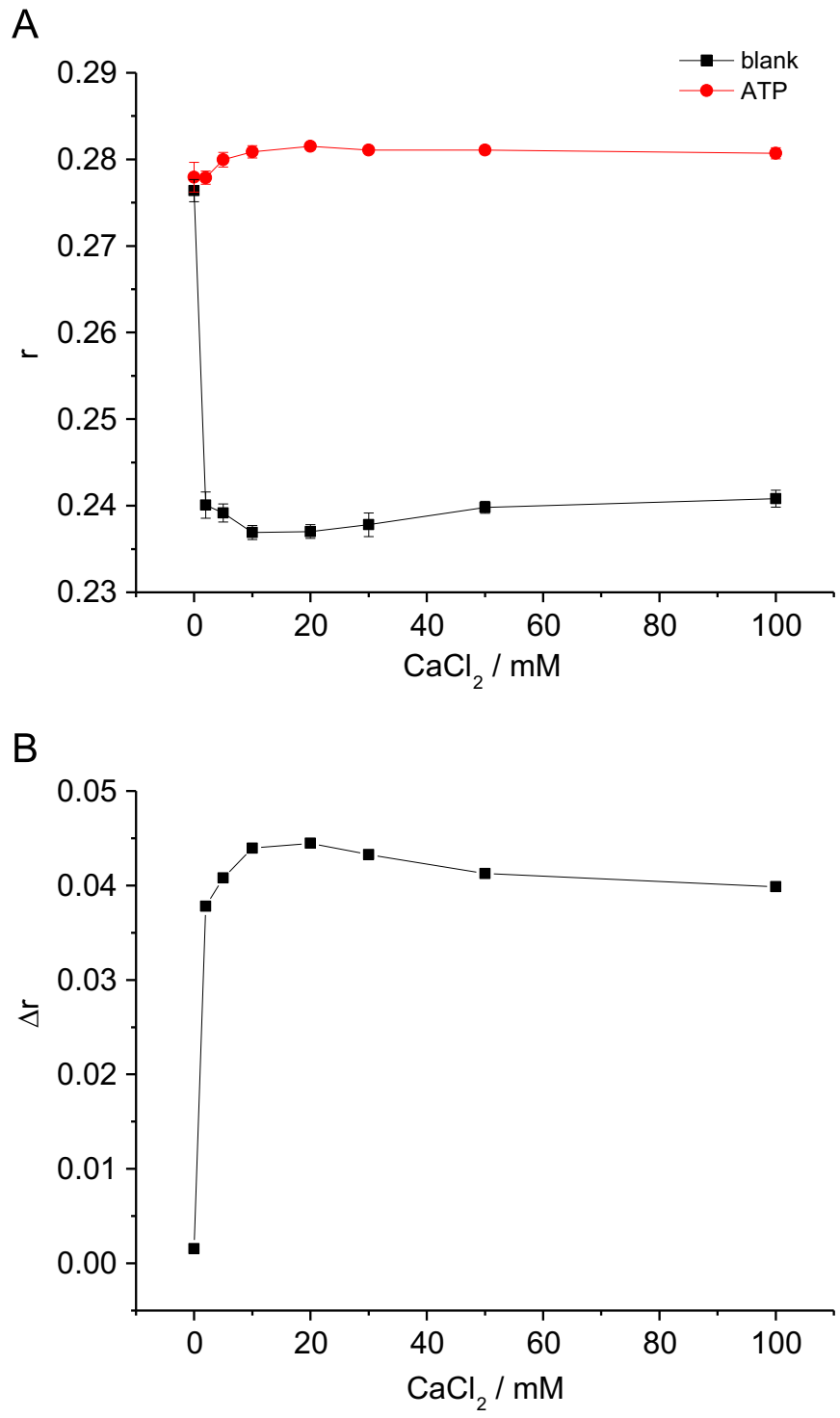

Fig. 3. Testing effect of $\mathrm{CaCl}_{2}$ on ATP-induced FA change of 23A-TMR-A25. (A) FA of 23A-TMR-A25 in absence of ATP or in presence of $100 \mu \mathrm{M}$ ATP was recorded in the binding buffer solution containing $50 \mathrm{mM}$ Tris- $\mathrm{HCl}(\mathrm{pH} 7.5), 50 \mathrm{mM} \mathrm{NaCl}, 0.1 \%$ Tween 20, and varying concentrations of $\mathrm{CaCl}_{2}$. (B) Net FA change caused by ATP versus varying concentrations of ATP.

2001; Lin and Patel, 1997). $20 \mathrm{mM} \mathrm{CaCl}_{2}$ was applied in the binding solution for further work.

The $\mathrm{pH}$ of buffer solution did not show great effect on the ATPinduced FA change of 23A-TMR-A25 in the range from 4.0 to 8.0, and the ATP-induced FA change began to decrease when $\mathrm{pH}$ was higher than 8.0 (Fig. S4). In further experiments binding buffer solution with $\mathrm{pH}$ at 7.5 was used. We also investigated the effect of incubation time on the FA change caused by ATP. Incubation of mixture solution for one minute allowed for a large FA change, and longer incubation time did not further increase the obtained FA change, suggesting the FA sensing process was rapid. 10-min incubation was applied to achieve enough equilibrium of binding.

Incubation temperature had great effect on sensing ATP with TMR-labeled aptamers. With temperature increasing, the FA of 23A-TMR-A25 in absence of ATP decreased as the rotation of the aptamer probe increased at high temperature. Temperature ranging from $20^{\circ} \mathrm{C}$ to $30^{\circ} \mathrm{C}$ was favorable for FA sensing with 23ATMR-A25 as larger ATP-induced FA change was achieved (Fig. S5). Temperature at $20^{\circ} \mathrm{C}$ was chosen for FA sensing of ATP in this work. The addition of $0.1 \%$ Tween 20 could help to reduce the nonspecific absorption of TMR-labeled aptamer. Finally, the binding buffer solution containing $50 \mathrm{mM}$ Tris- $\mathrm{HCl}$ (pH 7.5), $50 \mathrm{mM}$ $\mathrm{NaCl}, 20 \mathrm{mM} \mathrm{CaCl}$, and $0.1 \%$ Tween 20 was used in FA sensing with 23A-TMR-A25, and incubation at $20^{\circ} \mathrm{C}$ for $10 \mathrm{~min}$ was applied.

The experimental condition for aptamer probe 16C-TMR-A25 to sense ATP was also optimized. The same binding buffer solution was favorable for 16C-TMR-A25 as that used for aptamer probe 23A-TMR-A25. $\mathrm{CaCl}_{2}$ had significant effect on the ATP-induced FA change (Fig. S6), while $\mathrm{NaCl}$ showed little effect. A fast FA response of 16 C-TMR-A25 to ATP also enabled the short incubation in FA analysis. High FA decrease of 16C-TMR-A25 caused by ATP was observed when solution $\mathrm{pH}$ ranged from 6.0 to 8.0 (Fig. S7). When pH was lower than 6.0 or higher than 8.0, the obtained FA change was lower. The result shows $\mathrm{pH}$ ranging from 6.0 to 8.0 is favorable in sensing ATP with 16C-TMR-A25. Low temperature did not significantly affect the FA response of 16C-TMR-A25 to ATP (Fig. S8). High temperature above $50{ }^{\circ} \mathrm{C}$ disrupted the capability of $16 \mathrm{C}$ TMR-A25 for FA sensing. Incubation at $20^{\circ} \mathrm{C}$ was also applied in sensing ATP with 16C-TMR-A25.

\subsection{FA sensing ATP}

At optimized experimental conditions, ATP was successfully analyzed with 23A-TMR-A25 or 16C-TMR-A25 aptamer FA sensor (Fig. 4). By using 23A-TMR-A25, FA-increasing response upon ATP was obtained. ATP ranging from $1 \mu \mathrm{M}$ to $200 \mu \mathrm{M}$ could be detected, and the maximum FA increase was about 0.047 . The detection limit reached $1 \mu \mathrm{M}$. The high FA increase is not attributed to the increase of molecular weight as ATP has small molecular weight (about $507 \mathrm{~g} / \mathrm{mole}$ ) and ATP binding only leads to small increase ( $\sim 6 \%$ increase) in molecular weight of the 23A-TMR-A25 (about $8395 \mathrm{~g} / \mathrm{mole}$ ). The fluorescence intensity of 23A-TMR-A25 decreased with increasing concentrations of ATP, and the maximum decrease in fluorescence intensity was about 39\% (data not shown). The decrease of fluorescence intensity indicates the strengthening of TMR-G interaction in aptamer upon ATP addition because TMR-G interaction can cause fluorescence quenching of TMR. Thus, the increase of FA of 23A-TMR-A25 is probably due to the enhancement of TMR-G interaction, decreasing the freedom of local rotation of labeled TMR. The dissociation constant $\left(K_{\mathrm{d}}\right)$ of 23A-TMR-A25 was estimated to be about $9 \mu \mathrm{M}$ by extracting the fraction of bound probe over the total concentration of the probe from FA responses of aptamer probe to varying concentrations of ATP and non-linear fitting (Jing and Bowser, 2011; Zhao et al., 2014). It is close to the reported $K_{\mathrm{d}}$ of ATP-binding aptamer (Deng et al., 2001; Huizenga and Szostak, 1995). The result shows the labeling of TMR on the 23rd A in the aptamer does not significantly affect the binding affinity of aptamer.

16C-TMR-A25 was used as a FA sensor with FA-decreasing response to detect ATP. ATP in the range from $1 \mu \mathrm{M}$ to $200 \mu \mathrm{M}$ could be detected with a detection limit of $1 \mu \mathrm{M}$. The obtained maximum absolute FA decrease of 16 C-TMR-A25 was about 0.052. The dramatic decrease of FA of 16C-TMR-A25 was due to the ATPbinding reduced TMR-G interaction, which increased the local rotation of TMR. The weaken interaction could be proved by the increase of fluorescence intensity of 16C-TMR-A25 (about 59\% maximum increase in fluorescence intensity) with increasing of ATP. The reduced interaction eliminated the quenching of TMR caused by $G$ bases, which is in agreement with previous studies (Heinlein et al., 2003; Unruh et al., 2005; Yabuki et al., 2003; Zhao et al., 2014). The $K_{\mathrm{d}}$ of 16C-TMR-A25 was estimated to be about $10 \mu \mathrm{M}$ (Jing and Bowser, 2011; Zhao et al., 2014), close to that of 23A-TMR-A25 and the reported $K_{\mathrm{d}}$ of aptamer for ATP (Deng, et al. 2001; Huizenga and Szostak, 1995). It shows TMR labeling in 


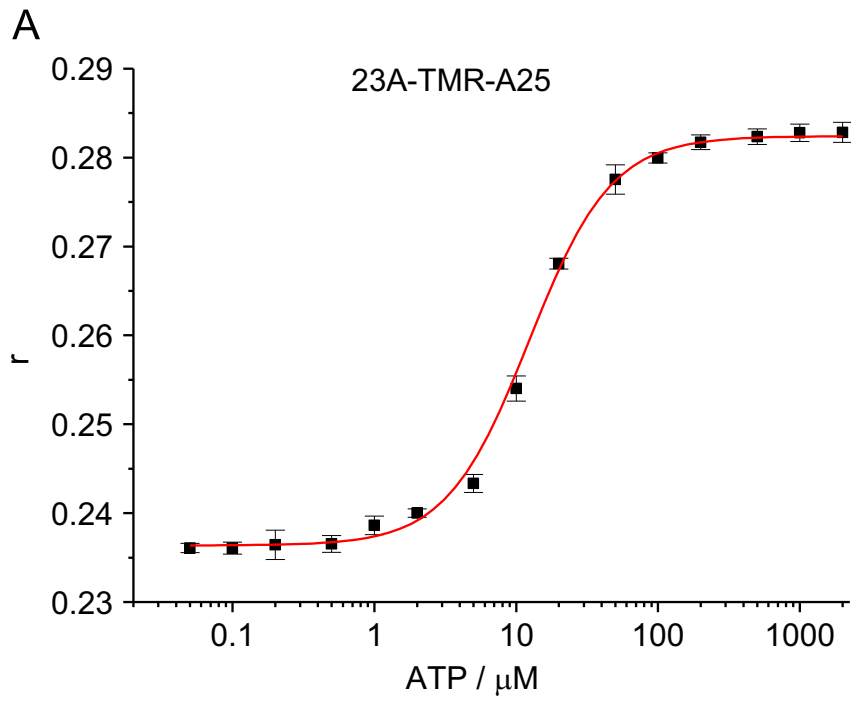

B

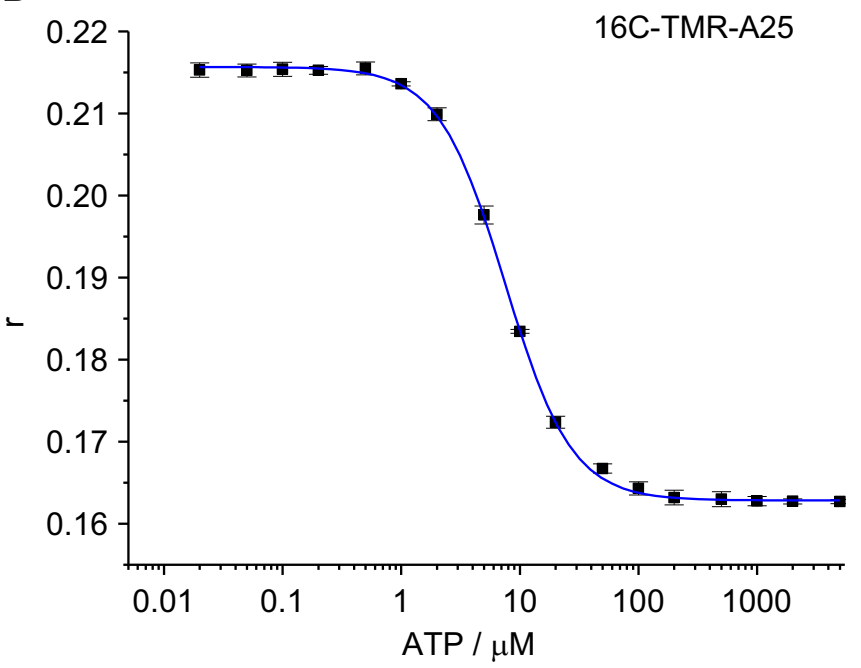

Fig. 4. FA sensing ATP with 23A-TMR-A25 (A) or 16C-TMR-A25 (B).

16C-TMR-A25 does not reduce the binding affinity of aptamer against ATP. 16C-TMR-A25 and 23A-TMR-A25 can be used as a pair of aptamer probes for FA sensing, showing complementary FA response to ATP. The obtained sensitivity for ATP analysis in this work is comparable to that obtained in many aptamer-based FA assays and fluorescence assays for ATP (Cui et al., 2012; Feng et al., 2014; Juskowiak, 2011; Liu et al., 2009, 2013; Sassolas et al., 2011; Zhu et al., 2012).

\subsection{Selectivity of FA sensors for ATP}

We assessed the selectivity of FA sensors of 23A-TMR-A25 and 16C-TMR-A25 by testing other small molecules including guanosine, thymidine, cytidine, GTP, CTP, and UTP (Fig. 5). All of the tested small molecules did not induce remarkable FA changes of 23A-TMR-A25 and 16C-TMR-A25. The simultaneous presence of the tested small molecules and ATP did not affect the detection of ATP. The results show 23A-TMR-A25 and 16C-TMR-A25 both exhibit good selectivity in analyzing ATP.

We further tested the feasibility of FA sensor for detection of ATP in complex sample matrix. ATP spiked in diluted human serum samples were analyzed by using 16C-TMR-A25 (Fig. S9). In 100-fold diluted human serum sample, the 16C-TMR-A25 aptamer probe showed the same FA response upon ATP as in binding buffer

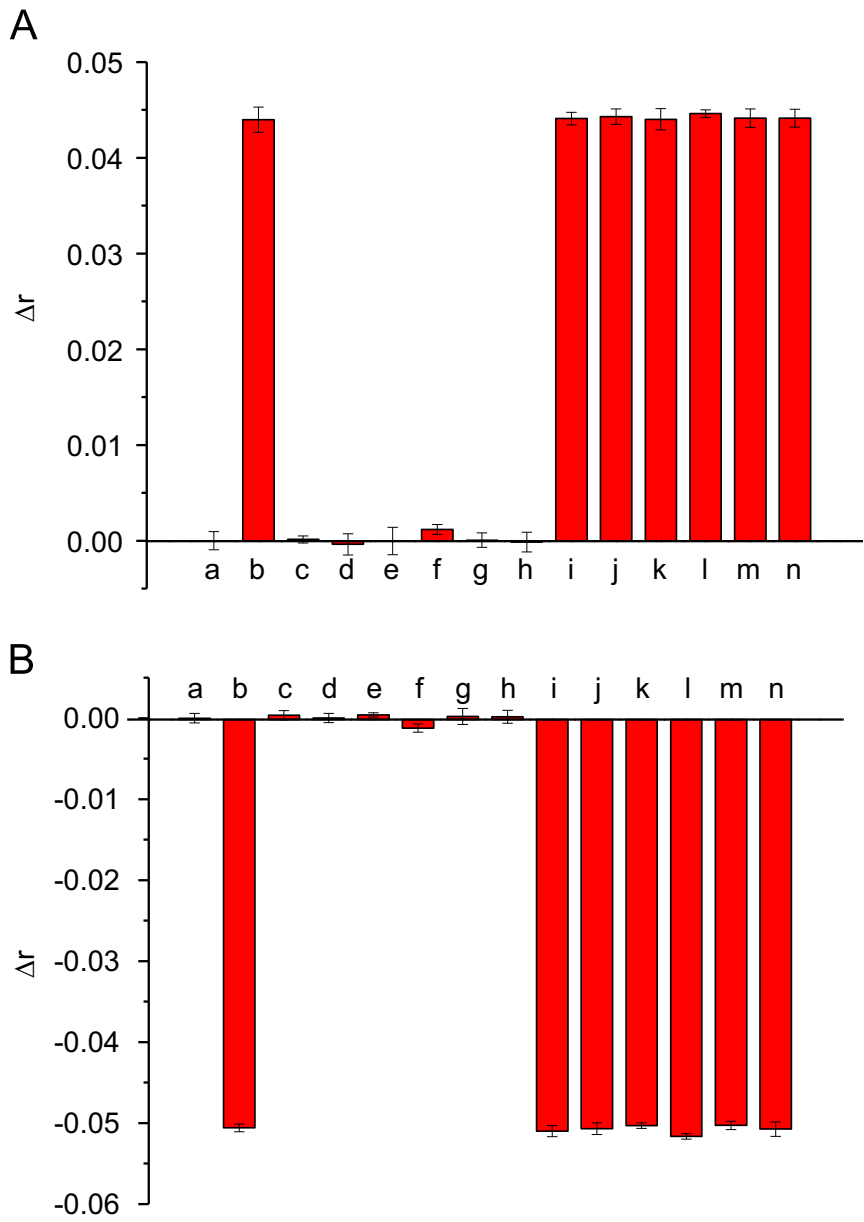

Fig. 5. Selectivity of FA sensing ATP with 23A-TMR-A25 (A) or 16C-TMR-A25 (B) aptamer probe. (a) blank; (b) $100 \mu \mathrm{M}$ ATP; (c) $2 \mathrm{mM}$ guanosine, (d) $2 \mathrm{mM}$ thymidine; (e) $2 \mathrm{mM}$ cytidine; (f) $0.2 \mathrm{mM}$ GTP; (g) $2 \mathrm{mM} \mathrm{CTP;} \mathrm{(h)} 2 \mathrm{mM}$ UTP; (i) $100 \mu \mathrm{M} \mathrm{ATP}+2 \mathrm{mM}$ guanosine, (j) $100 \mu \mathrm{M}$ ATP $+2 \mathrm{mM}$ thymidine; (k) $100 \mu \mathrm{M}$ ATP +2 mM cytidine; (l) $100 \mu \mathrm{M}$ ATP $+0.2 \mathrm{mM}$ GTP; (m) $100 \mu \mathrm{M}$ ATP $+2 \mathrm{mM}$ CTP; (n) $100 \mu \mathrm{M}$ ATP $+2 \mathrm{mM}$ UTP.

solution, and the detection limit of ATP still reached $1 \mu \mathrm{M}$. In 20fold diluted human serum sample 16C-TMR-A25 allowed the detection of spiked ATP as low as $2 \mu \mathrm{M}$. 23A-TMR-A25 probe also enabled the successful detection of ATP spiked in diluted human serum sample. ATP as low as $1 \mu \mathrm{M}$ could be detected in 20 -fold diluted human serum sample or 100 -fold diluted serum sample by using 23A-TMR-A25 (Fig. S9). These results show the 16C-TMRA25 and 23A-TMR-A25 aptamer probes both can be applied to detect ATP in complex sample matrix.

\section{Conclusion}

We achieved comprehensive mapping the labeling sites (A, C, T bases, and terminals) of the anti-ATP aptamer to construct fluorescence anisotropy sensors for ATP detection with TMR-labeled aptamers. The FA sensing was based on the ATP-binding induced change of intramolecular interaction between TMR and G bases of aptamers and alteration of FA of labeled TMR. By screening 14 labeling positions, favorable aptamer probes with remarkable FAincrease or FA-decrease response to ATP were obtained when TMR was labeled on 16th $C$ or 23rd A of the aptamer. These aptamer probes allowed detecting ATP at $1 \mu \mathrm{M}$ with good selectivity. Full screening labeling sites of aptamers (A, C, T bases and terminal ends) greatly enhances the probability to build a TMR-labeled 
aptamer sensor with remarkable FA response upon target-binding. This success will widen the application of aptamer-based FA sensor relying on interaction between TMR and G bases and other aptamer-based fluorescence approach in target analysis.

\section{Acknowledgment}

The work was supported from the National Natural Science Foundation of China (Grant no. 21222503, 21435008), Outstanding Youth Talents Program of Shanxi Province, and the Key Project of Chinese Ministry of Education (Grant no. 212020).

\section{Appendix A. Supplementary material}

Supplementary data associated with this article can be found in the online version at http://dx.doi.org/10.1016/j.bios.2015.03.031.

\section{References}

Citartan, M., Gopinath, S.C.B., Tominaga, J., Tan, S.-C., Tang, T.-H., 2012. Biosens. Bioelectron. 34, 1-11.

Cruz-Aguado, J.A., Penner, G., 2008. Anal. Chem. 80, 8853-8855.

Cui, L., Zou, Y., Lin, N.H., Zhu, Z., Jenkins, G., Yang, C.Y., 2012. Anal. Chem. 84, $5535-5541$.

Deng, Q., German, I., Buchanan, D., Kennedy, R.T., 2001. Anal. Chem. 73, 5415-5421.

Feng, C., Dai., S., Wang, L., 2014. Biosens. Bioelectron. 59, 64-74.

Gokulrangan, G., Unruh, J.R., Holub, D.F., Ingram, B., Johnson, C.K., Wilson, G.S., 2005. Anal. Chem. 77, 1963-1970.

Gradinaru, C.C., Marushchak, D.O., Samim, M., Krull, U.J., 2010. Analyst 135, $452-459$
Heinlein, T., Knemeyer, J.P., Piestert, O., Sauer, M., 2003. J. Phys. Chem. B 107, 7957-7964.

Huang, Y., Zhao, S., Chen, Z.F., Shi, M., Liang, H., 2012. Chem. Commun. 48, 7480-7482.

Huizenga, D.E., Szostak, J.W., 1995. Biochemistry 34, 656-665.

Jing, M., Bowser, M.T., 2011. Anal. Chim. Acta 686, 9-18.

Juskowiak, B., 2011. Anal. Bioanal. Chem. 399, 3157-3176.

Kidd, A., Guieu, V., Perrier, S., Ravelet, C., Peyrin, E., 2011. Anal. Bioanal. Chem. 401, 3229-3234.

Kim, Y.S., Gu, M.B., 2014. Adv. Biochem. Eng. Biotechnol. 140, 29-67.

Knowles, J.R., 1980. Annu. Rev. Biochem. 49, 877-919.

Le, X.C., Wan, Q.H., Lam, M.T., 2002. Electrophoresis 23, 903-908.

Lea, W.A., Simeonov, A.W., 2011. Expert Opin. Drug Discov. 6, 17-32.

Li, F., Zhang, H., Wang, Z., Newbigging, A.M., Reid, M.S., Li, X.F., Le, X.C., 2015. Anal. Chem. 87, 274-292.

Lin, C.H., Patel, D.J., 1997. Chem. Biol. 4, 817-832.

Liu, J., Cao, Z., Lu, Y., 2009. Chem. Rev. 109, 1948-1998.

Liu, J., Wang, C., Jiang, Y., Hu, Y., Li, J., Yang, S., Li, Y., Yang, R., Tan, W., Huang, C.Z., 2013. Anal. Chem. 85, 1424-1430.

McKeague, M., Derosa, M.C., 2012. J. Nucleic Acids, 748913.

Perrier, S., Ravelet, C., Guieu, V., Roy, B., Perigaud, C., Peyrin, E., 2010. Biosens. Bioelectron. 25, 1652-1657.

Ruta, J., Perrier, S., Ravelet, C., Fize, J., Peyrin, E., 2009. Anal. Chem. 81, 7468-7473. Sassolas, A., Blum, L.J., Leca-Bouvier, B.D., 2011. Analyst 136, 257-274.

Smith, D.S., Eremin, S.A., 2008. Anal. Bioanal. Chem. 391, 1499-1507.

Unruh, J.R., Gokulrangan, G., Wilson, G.S., Johnson, C.K., 2005. Photochem. Photobiol. 81, 682-690.

Yabuki, K., Ouchi, T., Ohya, Y., 2003. Supramol. Chem. 15, 149-154.

Ye, B.C., Yin, B.C., 2008. Angew Chem. Int. Ed. 47, 8386-8389.

Yu, Y., Liu, Y., Zhen, S.J., Huang, C.Z., 2013. Chem. Commun. 49, 1942-1944.

Zhang, D., Lu, M., Wang, H., 2011. J. Am. Chem. Soc. 133, 9188-9191.

Zhang, D., Zhao, Q., Zhao, B., Wang, H., 2012. Anal. Chem. 84, 3070-3074.

Zhao, Q., Lv, Q., Wang, H., 2014. Anal. Chem. 86, 1238-1245.

Zhu, Z., Ravelet, C., Perrier, S., Guieu, V., Fiore, E., Peyrin, E., 2012. Anal. Chem. 84, 7203-7211.

Zhu, Z., Schmidt, T., Mahrous, M., Guieu, V., Perrier, S., Ravelet, C., Peyrin, E., 2011. Anal. Chim. Acta 707, 191-196. 